\title{
In Vivo Attenuation of M-Current Suppression Impairs Consolidation of Object Recognition Memory
}

\author{
Anastasia Kosenko, ${ }^{1}$ Shirin Moftakhar, ${ }^{1}$ Marcelo A. Wood, ${ }^{2}$ and Naoto Hoshi ${ }^{1,3,4}$ \\ ${ }^{1}$ Department of Pharmacology, University of California, Irvine, Irvine, California 92697, ${ }^{2}$ Department of Neurobiology and Behavior, Center for the \\ Neurobiology of Learning and Memory, University of California, Irvine, Irvine, California 92697, ${ }^{3}$ Department of Pharmaceutical Sciences, \\ University of California, Irvine, Irvine, California 92617, and ${ }^{4}$ Department of Physiology and Biophysics, University of California, Irvine, Irvine, \\ California 92697
}

The M-current is a low voltage-activated potassium current generated by neuronal Kv7 channels. A prominent role of the Mcurrent is to a create transient increase of neuronal excitability in response to neurotransmitters through the suppression of this current. Accordingly, M-current suppression is assumed to be involved in higher brain functions including learning and memory. However, there is little evidence supporting such a role to date. To address this gap, we examined behavioral tasks to assess learning and memory in homozygous Kv7.2 knock-in mice, Kv7.2(S559A), which show reduced M-current suppression while maintaining a normal basal M-current activity in neurons. We found that Kv7.2(S559A) mice had normal object location memory and contextual fear memory, but impaired long-term object recognition memory. Furthermore, short-term memory for object recognition was intact in Kv7.2(S559A) mice. The deficit in long-term object recognition memory was restored by the administration of a selective $\mathrm{Kv7}$ channel inhibitor, XE991, when delivered during the memory consolidation phase. Lastly, c-Fos induction $2 \mathrm{~h}$ after training in Kv7.2(S559A) mice was normal in the hippocampus, which corresponds to intact object location memory, but was reduced in the perirhinal cortex, which corresponds to impaired long-term object recognition memory. Together, these results support the overall conclusion that M-current suppression is important for memory consolidation of specific types of memories.

Key words: KCNQ2; Kv7.2; object location memory; object recognition memory; potassium channel; voltage-gated channel

Significance Statement

Dynamic regulation of neuronal excitation is a fundamental mechanism for information processing in the brain, which is mediated by changes in synaptic transmissions or by changes in ion channel activity. Some neurotransmitters can facilitate action potential firing by suppression of a low voltage-activated potassium current, M-current. We demonstrate that M-current suppression is critical for establishment of long-term object recognition memory, but is not required for establishment of hippocampus-dependent location memory or contextual memory. This study suggests that M-current suppression is important for stable encoding of specific types of memories.

\section{Introduction}

Neuronal Kv7 channels are low voltage-gated potassium channels that are widely expressed in both central and peripheral neurons and generate a current commonly known as the M-current (Jentsch, 2000; Delmas and Brown, 2005; Greene and Hoshi,

Received Feb. 13, 2020; revised May 8, 2020; accepted June 6, 2020.

Author contributions: A.K., M.A.W., and N.H. designed research; A.K., S.M., and N.H. performed research;

S.M. and M.A.W. contributed unpublished reagents/analytic tools; A.K., S.M., and N.H. analyzed data; A.K. and N.H. wrote the paper.

This work was partially supported by National Institutes of Neurological Disorders and Stroke Grant R01NS-067288 to N.H.

A. Kosenko's present address: Bristol Myers Squibb, Redwood City, CA 94063.

The authors declare no competing financial interests.

Correspondence should be addressed to Naoto Hoshi at nhoshi@uci.edu.

https://doi.org/10.1523/JNEUROSCI.0348-20.2020

Copyright $\odot 2020$ the authors
2017). Neuronal Kv7 channels are encoded by subclasses of the KCNQ gene family, KCNQ2, 3, 4, and 5. Since neuronal Kv7 channels are activated near the resting membrane potential, the M-current stabilizes membrane potential at the resting potential and controls neuronal excitability including frequency of action potential firing (Jentsch, 2000; Delmas and Brown, 2005). Not surprisingly, mutations in KCNQ gene family have been identified in various forms of epilepsy and encephalopathy (Miceli et al., 2010, 2014).

An additional important function of the M-current is to reversibly increase neuronal excitability via suppression of the $\mathrm{M}$-current in response to activation of Gq-coupled receptors such as $\mathrm{m} 1$ muscarinic acetylcholine receptor (Delmas and Brown, 2005; Greene and Hoshi, 2017). Because of this dynamic regulatory role on neuronal excitability, M-current suppression 
has been implicated in higher brain functions such as attention and memory (Delmas and Brown, 2005). However, there is little experimental evidence supporting this function. Our previous cell-based studies show that an alanine substitution of the conserved protein kinase C (PKC) phosphorylation acceptor site in Kv7.2 subunit, which corresponds to serine 559 in mouse orthologue mKv7.2(S559A), removes most of M-current suppression (Hoshi et al., 2003, 2005, 2010; Kosenko et al., 2012; Kay et al., 2015). To elucidate roles of M-current suppression in higher brain function, we recently generated Kv7.2(S559A) knock-in mice (Greene et al., 2018). Kv7.2(S559A) mice showed significantly attenuated M-current suppression but had normal basal M-current density (Greene et al., 2018). Our previous study shows that Kv7.2(S559A) mice were resistant to chemoconvulsants and showed minimal neuronal death after status epilepticus (Greene et al., 2018), which suggested a pathologic role of M-current suppression in seizures. In this present study, we analyzed performance of homozygous Kv7.2(S559A) knock-in mice in behavioral learning tasks to determine whether M-current suppression plays a role in memory processing.

\section{Materials and Methods}

Mice

Mice were either C57BL/6J or Kv7.2(S559A) knock-in mice in C57BL/6J background. Generation of Kv7.2(S559A) mice has been described (Greene et al., 2018). All experiments were conducted according to US National Institutes of Health guidelines for animal care and use and were approved by the Institutional Animal Care and Use Committee of the University of California, Irvine.

\section{Primary neuron culture}

Neuron isolation and primary neuron culture were performed according to the protocol described previously (Kaech and Banker, 2006) with some modifications. Briefly, the cortices of neonatal pups from wildtype (WT) or homozygous Kv7.2(S559A) mice were treated with papain, and DNase and plated onto poly-D-lysine-coated $35 \mathrm{~mm}$ dishes. Cultured cells were maintained in a medium (Neurobasal A, B27, GlutaMAX, and $5 \mu \mathrm{M}$ AraC).

\section{Experimental design and statistical analysis}

Electrophysiological measurements. Perforated patch-clamp recordings were performed at room temperature on isolated primary cortical neurons between 13 and $15 \mathrm{~d}$ in vitro from wild-type and homozygous Kv7.2(S559A) mice using an Axopatch 200B patch-clamp amplifier (Molecular Devices) as described previously (Hoshi et al., 2005; Kay et al., 2015; Greene et al., 2018). Signals were sampled at $2 \mathrm{kHz}$, filtered at $1 \mathrm{kHz}$, and acquired using pClamp software (version 10; Molecular Devices). Patch pipettes (3-4 M $\Omega$ ) were filled with an intracellular solution containing $135 \mathrm{~mm}$ potassium acetate, $15 \mathrm{~mm} \mathrm{KCl,} 3 \mathrm{~mm} \mathrm{MgCl}$, 6 $\mathrm{mm} \mathrm{NaCl}, 10 \mathrm{~mm}$ HEPES, pH 7.2, and amphotericin B (0.1-0.2 mg/ml). The extracellular solution consisted of $130 \mathrm{~mm} \mathrm{NaCl}, 3 \mathrm{~mm} \mathrm{KCl}, 1 \mathrm{~mm}$ $\mathrm{MgCl}_{2}, 0.1 \mathrm{~mm} \mathrm{CaCl}, 11 \mathrm{~mm}$ glucose, and $10 \mathrm{~mm}$ HEPES, pH 7.4 with $\mathrm{NaOH}$. Amplitudes of the M-currents were measured as XE991-sensitive standing currents at $-30 \mathrm{mV}$. For current-clamp recording, membrane potentials were adjusted to $-70 \mathrm{mV}$ and minimal $500 \mathrm{~ms}$ current injection in a $5 \mathrm{pA}$ increment that induced action potential firing with more than one action potential was used to monitor neuronal excitability using a sampling frequency of $5 \mathrm{kHz}$.

Behavioral analyses. All mice used in the behavioral experiments were between 8 and 15 weeks old at the time of testing. Both sexes were used except for odor recognition tests, where only males were used. All mice had ad libitum access to food and water, unless stated otherwise. The lights were maintained on a $12 \mathrm{~h}$ light/dark cycle, and all behavioral experiments were performed during the light cycle with a consistent starting time between the groups. All mice were group housed, with a maximum of 1 week of single housing if required by the experimental protocol.
Open field tests. Locomotion was monitored in an automated activity system $(50 \times 50 \times 40 \mathrm{~cm})$ equipped with infrared sensors for both horizontal and vertical activity measurements (VersaMax, AccuScan Instruments). Exploratory behavior was monitored during the first $30 \mathrm{~min}$ without prior habituation to the testing arena. Data were collected using VersaMax software (AccuScan). Distance traveled and duration of mice located were recorded and analyzed as described previously (Xu et al., 2004; Okamura et al., 2008).

Rotarod test. The rotarod test was conducted as described previously (Duangdao et al., 2009). Mice were placed on a rotarod (TSE Systems) and the latency and rotation speed, at which the mice fell off the rod, were recorded automatically by an infrared beam located below the rotating rod. Each mouse was given three trials with intertrial intervals of $20 \mathrm{~min}$.

Object location memory and object recognition memory tasks. Object location memory (OLM) and object recognition memory (ORM) tasks were conducted as previously described with minor modification (Vogel-Ciernia and Wood, 2014; Kwapis et al., 2019). Homozygous Kv7.2(S559A) and wild-type littermates were used in all behavioral experiments. All mice were handled for $1 \mathrm{~min} / \mathrm{d}$ for $3 \mathrm{~d}$, followed by $3 \mathrm{~d}$ of habituation to the testing arena for $5 \mathrm{~min} / \mathrm{d}$. During the training session, two identical objects were placed and each mouse was allowed to explore the objects for $10 \mathrm{~min}$. For the test session, one object was moved to a new location (OLM) or one object was replaced with a novel object (ORM). Mice were allowed to explore the objects for $5 \mathrm{~min}$, and mouse movements were video recorded. The time spent exploring each object was manually scored by experimenters blind to experimental groups. Mice that explored both objects for $<3 \mathrm{~s}$ in either training or test sessions were excluded from further analyses. Preference for the novel item was expressed as a discrimination index [DI $=\left(t_{\text {novel }}-t_{\text {familiar }}\right) /\left(t_{\text {novel }}+\right.$ $\left.\left.t_{\text {familiar }}\right) \times 100\right]$.

For XE991 rescue experiments, XE991 (2 mg/kg, i.p.) in PBS or PBS vehicle (veh) control were administered $15 \mathrm{~min}$ before training, immediately after training, or $15 \mathrm{~min}$ before test session.

Social olfactory memory task. Olfactory memory experiments were conducted using single-housed male mice as described (Feinberg et al., 2012). The social odor stimuli were presented on wooden beads (WoodWorks) scented by adult male odor donor mice. During the training session, mice were allowed to explore a single bead scented with a novel social odor for $2 \mathrm{~min}$. Twenty minutes or $24 \mathrm{~h}$ following training, experimental mice were presented with two beads, one scented with the social odor presented during training and the other scented with a novel social odor. Mice were allowed to explore the beads for $2 \mathrm{~min}$ after the initiation of exploration. Sniffing was scored manually and expressed as the discrimination index.

Test of olfactory function. Mice were single housed for 3 consecutive days before the test. During this time, a piece of cookie, Teddy Grahams (Nabisco), was placed into the cages of subject mice every $24 \mathrm{~h}$ to familiarize the mice with the odor and flavor of the cookie. Mice were fasted for $24 \mathrm{~h}$ before the test. During the test, the mouse was placed into a clean cage with $3 \mathrm{~cm}$ of clean bedding and allowed to acclimate to the cage for $5 \mathrm{~min}$. The mouse was then transferred into another clean cage and a food stimulus (cookie) was buried $\sim 1 \mathrm{~cm}$ beneath the bedding surface, in a random corner of the cage. The observer started the stopwatch when the mouse was reintroduced into the cage and stopped it when the mouse found the buried cookie. The test was discontinued if the mouse did not find the food within $15 \mathrm{~min}$.

Odor habituation/dishabituation task. This test consists of the sequential presentation of different odors and assesses the ability of mice to smell and distinguish same and different odors. The following sequence of odors was used: water - almond - banana - social odor 1 social odor 2. Each odor was presented in three consecutive trials for a duration of $2 \mathrm{~min}$ with an intertrial interval of $1 \mathrm{~min}$. Habituation is defined by a progressive reduction in olfactory investigation after repeated presentations of the same stimulus. Dishabituation is defined by the reinstatement of sniffing when a novel odor is presented. Odors were presented on cotton-tipped wooden applicators prepared on the day of the experiment. Nonsocial odors were obtained from pure natural almond and banana extracts (Frontier Natural Products), and social 
A

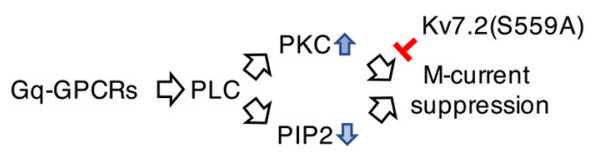
mKV7.2 534 TPGLKVSIRAVCVMRFLVSKRKFKEESLRPYDV 565 rKV7.2 516 TPGLKVSIRAVCVMRFLVSKRKFKESLRPYDV 547 hKV7.1507 REHHRATIKVIRRMQY FVAKKKFQQARKPYDV 538 hKV7.2 533 TPGLKVSIRAVCVMRFLVSKRKFKESLRPYDV 564 hKV7.3 512 IPTLKAAIRAVR ILQFRLYKKKKFKETLRPYDV 543 hKV7.4 527 MPAVKTVIRS IRILKFLYAKRKFKET hKV7.5 515 TPPLKTVIRAIRIMKFHVAKRKFKETLRPYDV 546

C
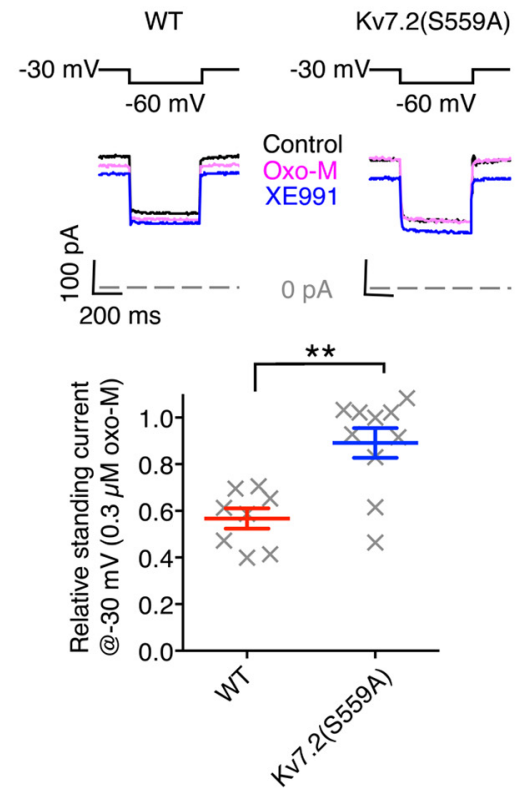

Mutated PKC phosphorylation site

B

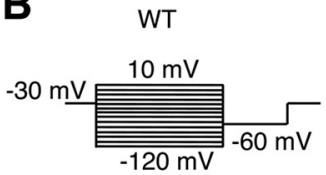

Kv7.2(S559A)
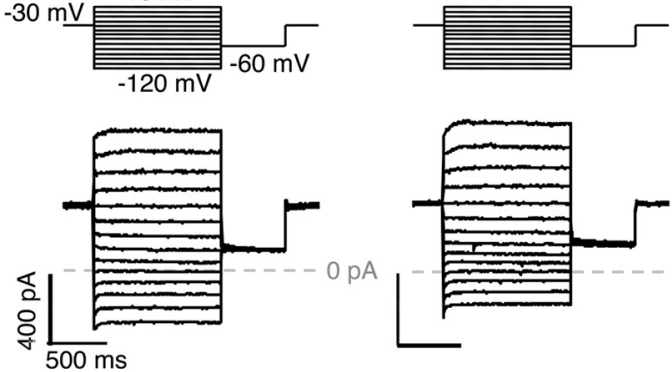

WT

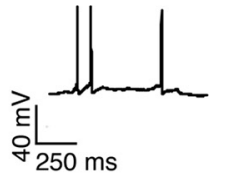

$1 \mu \mathrm{M}$ oxo

$10 \mu \mathrm{M}$ oxo

$\mathbf{F}$
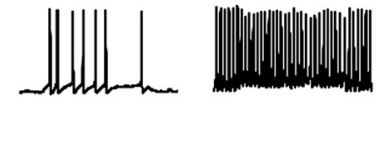

G

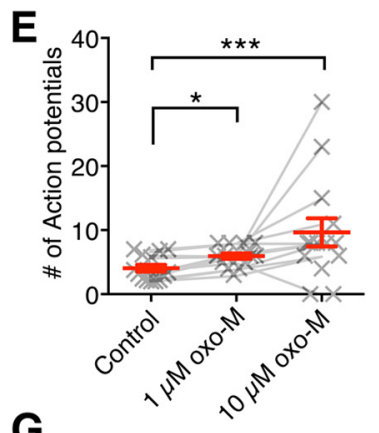

$1 \mu \mathrm{M}$ oxo

$10 \mu \mathrm{M}$ oxo
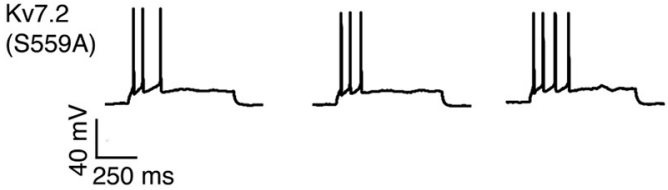

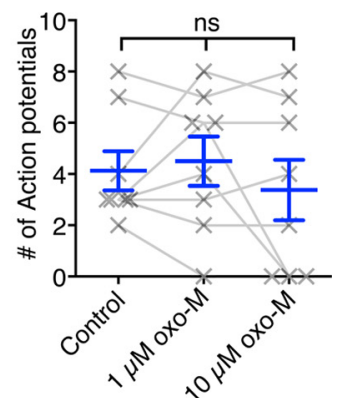

Figure 1. Cortical neurons from Kv7.2(S559A) mice showed attenuated M-current suppression induced by oxotremorine-M and lack of a transient increase in neuronal excitability. A, Schematic diagram of the pathway of M-current suppression and the point of intervention. Stimulation of $\mathrm{G}_{\mathrm{q}}-$ GPCRs activates phospholipase C (PLC), which consumes phosphatitylbisphosphate (PIP2) and activates PKC. Note that PKC mediating KV7.2 phosphorylation requires to be anchored to the Kv7.2 subunit via AKAP79/150 (see text for detail). Bottom, Aligned Kv7 channel protein sequences surrounding the mutated PKC acceptor site. $B$, Representative voltage-clamp current traces from cortical neurons showing $I-V$ responses. Voltage protocol is indicated at the top. C, M-current suppression by $0.3 \mu \mathrm{m}$ oxo-M suppression and complete inhibition by $20 \mu \mathrm{M}$ XE991 from cortical neurons of WT control and Kv7.2(S559A) mice. Voltage protocol (top), representative voltage-clamp traces (middle), and relative M-current suppression (bottom) are shown. $\boldsymbol{D}$, Representative voltage traces of current-clamp recording from cortical neurons of WT mice showing action potential firing in response to $500 \mathrm{~ms}$ current injections during control, and in the presence of $1 \mu \mathrm{m}$ oxo-M and $10 \mu \mathrm{m} \mathrm{oxo-M}$. E, Summary of change in neuronal excitability of wild-type cortical neurons in experiments shown in $\boldsymbol{D}$. $\boldsymbol{F}$, Representative voltage traces of current-clamp experiments from cortical neurons of Kv7.2(S559A) mice in the identical experiments shown in $\boldsymbol{D}$. G, Summary of change in neuronal excitability of Kv7.2(S559A) cortical neurons. Each data point represents different neuron. Results are shown as the mean \pm SEM. ns, $p>0.05 ;{ }^{*} p<0.01 ;{ }^{* *} p<0.01 ;{ }^{* * *} p<0.001$.

odors were obtained from mouse cages of the same sex as the subject mouse. The subject mouse was placed into a clean cage containing bedding for $30 \mathrm{~min}$, and a dry cotton-tipped applicator was inserted into the cage lid for habituation. Following the 30 min habituation, the testing trial began by inserting the applicator dipped into one of the odors into the cage lid. For each subsequent trial, the applicator was anchored to the lid at the same angle and to the same depth $(\sim 2.5 \mathrm{~cm})$. Sniffing was scored when the animal was orienting toward the applicator with its nose $2 \mathrm{~cm}$ or closer.

Contextual fear conditioning. Mice were handled for 3 consecutive days for $1 \mathrm{~min}$ each day. On the training day, mice were put into a conditioning chamber. A single $2 \mathrm{~s} 0.7 \mathrm{~mA}$ scrambled footshock was delivered at time 2 minutes and 28 seconds of a 3 min training period. A 5 min testing period, in which the mice were exposed to the same conditioned context in the absence of a shock, followed $24 \mathrm{~h}$ later. Freezing was
Table 1. Summary of electrophysiological property of cortical neurons

\begin{tabular}{lcc}
\hline & WT & \multicolumn{1}{c}{ Kv7.2(S559A) } \\
\hline Cell capacitance (pF) & $44.0 \pm 3.6(31)$ & $46.3 \pm 4.0(24)$ \\
Input resistance at $-70 \mathrm{mV}(\mathrm{M} \Omega)$ & $221 \pm 20.1(31)$ & $200.6 \pm 19.6(24)$ \\
Standing current at $-30 \mathrm{mV}(\mathrm{pA})$ & $362.4 \pm 50.0(9)$ & $328.7 \pm 32.0(10)$ \\
XE991-sensitive current at $-30 \mathrm{mV}(\mathrm{pA})$ & $90.5 \pm 24.6(9)$ & $71.2 \pm 11.1(10)$ \\
\hline
\end{tabular}

measured every $10 \mathrm{~s}$ for the duration of a 5 min test trial by an observer blind to the genotype. Freezing was measured and compared between the genotypes at the baseline, before the footshock, and $24 \mathrm{~h}$ following the footshock.

Immunohistochemistry for c-Fos. Two hours after training, mice were anesthetized with isoflurane and perfused with $4 \%$ paraformaldehyde 
(PFA) in PBS. After overnight postfixation in 4\% PFA, the brains were sectioned into $30-\mu \mathrm{m}$-thick coronal slices on a vibratome (VT1200, Leica). Immunohistochemistry was performed according to the freefloating method as described previously (Kay et al., 2015; Greene et al., 2018) with a rabbit polyclonal antibody against c-fos at 1:5000 dilution (Ab-5, Calbiochem) and Cy3-conjugated goat anti-rabbit IgG antibody at 1:500 dilution (Millipore). Immunofluorescent images were acquired using a fluorescent light microscope (DM4000B, Leica) equipped with a CCD camera (Optronics MicroFire, OPTMIF, Optronics). c-Fos-positive cells were defined as cells with four times stronger Cy3 fluorescent signals above tissue background. Positive cells were identified using a binary filter function in MetaMorph (Molecular Devices).

Statistical analysis. Mann-Whitney test was used for two comparison groups. Friedman test followed by Dunn's multiple-comparisons test was used for more than two comparison groups with repeated measures. Two-way ANOVA followed by Tukey's multiple-comparisons test was used to evaluate effects of genotypes and treatments. All analyses were two tailed, and the $\alpha$ value was set to 0.05 for significance. All statistical calculations were performed using Prism 6 (GraphPad Software). Error bars show the SEM.

\section{Results}

Cortical neurons from Kv7.2(S559A) mice showed normal M-current density with decreased M-current suppression and lack of neuronal hyperexcitation

To diminish M-current suppression in vivo, we generated Kv7.2 (S559A) knock-in mice as described in our previous study (Greene et al., 2018). The mutation introduced in Kv7.2 is an alanine substitution of serine 559, which is a conserved PKC phosphorylation acceptor residue critical for M-current suppression (Fig. 1A,B; Hoshi et al., 2003, 2005; Kosenko et al., 2012; Greene et al., 2018). Consistent with our previous findings in hippocampal and sympathetic ganglion neurons (Greene et al., 2018), cortical neurons from homozygous Kv7.2(S559A) knock-in mice showed no detectable changes in $I-V$ relationships (Fig. $1 B$ ), basic electrophysiological properties, and XE991-sensitive currents (Table 1). XE991 is a selective inhibitor of Kv7 channels.

Next, we examined M-current suppression induced by a muscarinic agonist, oxotremorine-M (oxo-M) in cortical neurons from homozygous Kv7.2(S559A) mice using voltage-clamp recordings. $\mathrm{M}$-current suppression induced by $0.3 \mu \mathrm{M}$ oxo-M was attenuated in cortical neurons from Kv7.2(S559A) mice (Fig. $1 C$; WT, $n=8$; Kv7.2(S559A), $n=10$, Mann-Whitney test, $p=0.004$ ), which was consistent with our previous study using hippocampal and sympathetic ganglion neurons (Greene et al., 2018). We examined modulation of neuronal excitability in cortical neurons induced by oxo-M using current-clamp analyses. Current-clamp recordings from wild-type mice showed increased neuronal firing when exposed to 1 or $10 \mu \mathrm{M}$ oxo-M during $500 \mathrm{~ms}$ depolarizing stimuli (Fig. $1 D, E ; n=13$, Friedman statistic $=14.51, p=0.0007)$. The $10 \mu \mathrm{M}$ oxo-M could induce spontaneous firing in wild-type cortical neurons (7 of 13 neurons; Fig. $1 D, E)$. Such change in neuronal excitability by oxo-M was not observed in cortical neurons from Kv7.2(S559A) mice (Fig. $1 F, G ; n=8$, Friedman statistic $=0.30, p=0.89$ ). Rather, 3 of 8 neurons showed complete suppression of action potential firing when $10 \mu \mathrm{M}$ oxo-M was applied (Fig. $1 G$ ). These results suggest that Kv7.2(S559A) mice have normal M-current with attenuated neuromodulation.

\section{Kv7.2(S559A) mice had normal exploratory behavior and motor coordination}

Since M-current suppression is implicated in various cognitive functions, including learning and memory, we hypothesized that attenuating M-current suppression by Kv7.2(S559A) mutation

\section{A Open field}

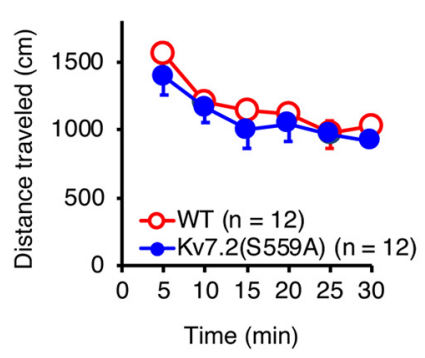

\section{Open field}

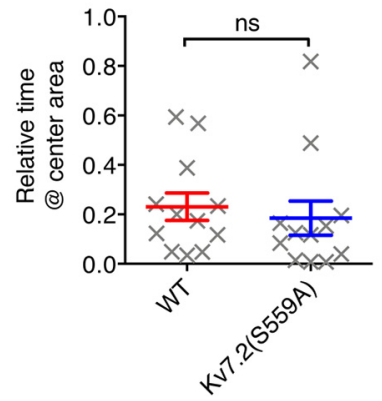

B Open field
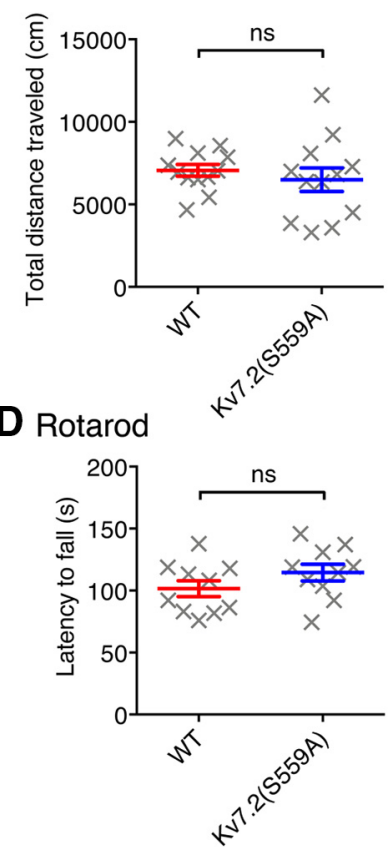

Figure 2. Kv7.2(S559A) mice had normal exploratory behaviors and motor coordination. $\boldsymbol{A}$, Exploratory activity during $30 \mathrm{~min}$ in an open field chamber. $\boldsymbol{B}$, Total distance traveled during experiments shown in $\boldsymbol{A}$. $\boldsymbol{C}$, Ratio of the time spent in the center of the arena over the time spent in the margins during a 5 min period in an open field chamber. $\boldsymbol{D}$, Latency to fall in rotarod test. Each data point represents a different animal. Results are shown as the mean \pm SEM, ns, $p>0.05$.

would impair some cognitive processes. Since many behavioral learning paradigms require normal exploratory behaviors and motor functions, we first conducted open field and rotarod tests to evaluate these functions. In open field tests, homozygous Kv7.2(S559A) and wild-type littermate control mice showed equivalent exploratory activities, as indicated by gradual habituation to an open field chamber (Fig. $2 A ; n=12$ /genotype), total travel distance (Fig. 2B; Mann-Whitney test, $p=0.35$ ), and time spent exploring the center area of the chamber (Fig. 2C; MannWhitney test, $p=0.22$ ). In rotarod tests, Kv7.2(S559A) mice and wild-type littermate controls showed similar latency to fall (Fig. $2 D ; n=10$ /genotype; Mann-Whitney test, $p=0.17$ ). These results suggest that Kv7.2(S559A) mice have normal motor coordination and exploratory behaviors.

\section{Kv7.2(S559A) mice had normal spatial memory and contextual fear memory}

To evaluate whether M-current suppression plays a role in spatial memory, we performed a standard OLM task (Vogel-Ciernia and Wood, 2014). Mice were placed in a context with two identical objects for $10 \mathrm{~min}$ during the training session, and then tested $24 \mathrm{~h}$ later in the same context with one of the objects moved to a novel location (Fig. 3A). Object location memory in this assay is indirectly determined from the discrimination index, which is defined as the time spent exploring the familiar object placed in a new location over the total time exploring both objects. Both Kv7.2(S559A) mice $(n=11)$ and wild-type littermate control mice $(n=12)$ showed similar exploration during training (Fig. $3 B$; Mann-Whitney test, $p=0.45$ ) as well as test sessions (Fig. $3 C$; Mann-Whitney test, $p=0.66$ ). In addition, both Kv7.2 (S559A) and wild-type littermate control mice showed increased 
A

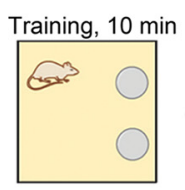

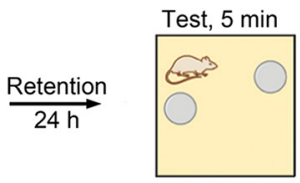

C
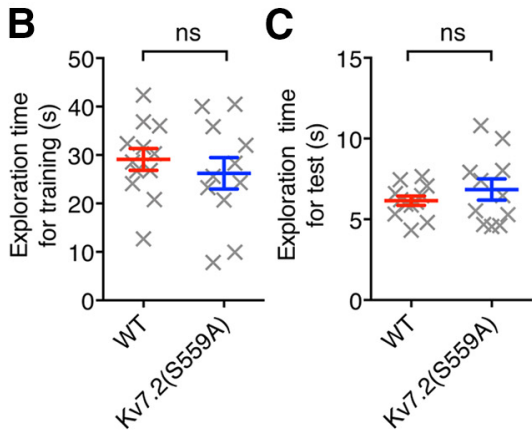
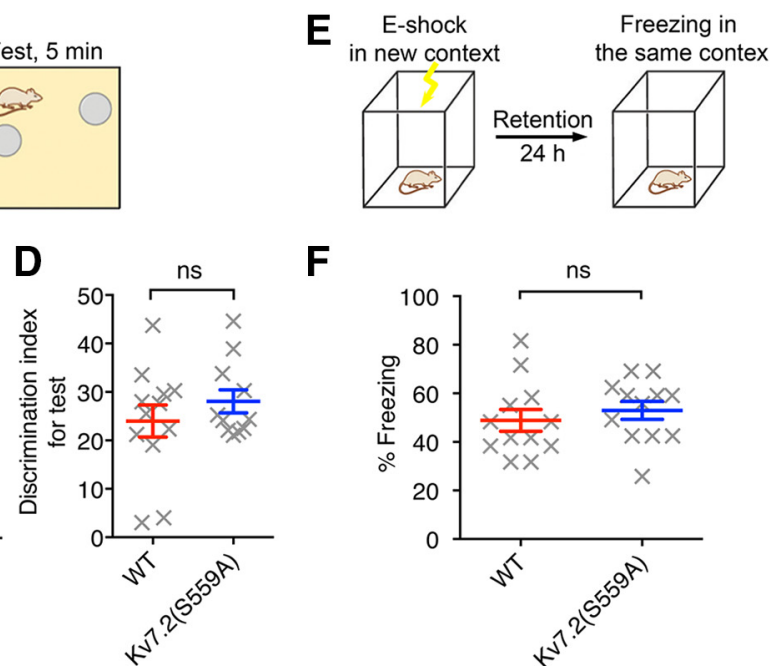

$\mathbf{F}$

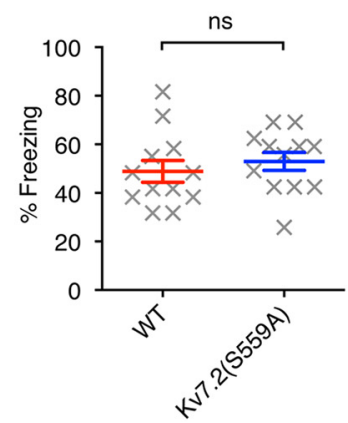

Figure 3. Kv7.2(S559A) mice had intact novel location recognition and contextual fear-conditioning memory. A, Schematic drawing showing the experimental design for object location tests. $\boldsymbol{B}$, Summary of total exploration time during a $10 \mathrm{~min}$ training session. C, Summary of total exploration time during 5 min test sessions. D, Summary of discrimination index in objection location test. $\boldsymbol{E}$, Schematic drawing showing the experimental design for contextual fear-conditioning tests. $\boldsymbol{F}$, Summary of freezing reactions in fear-conditioning tests. Each data point represents a different animal. Results are shown as the mean \pm SEM. $n s, p>0.05$.

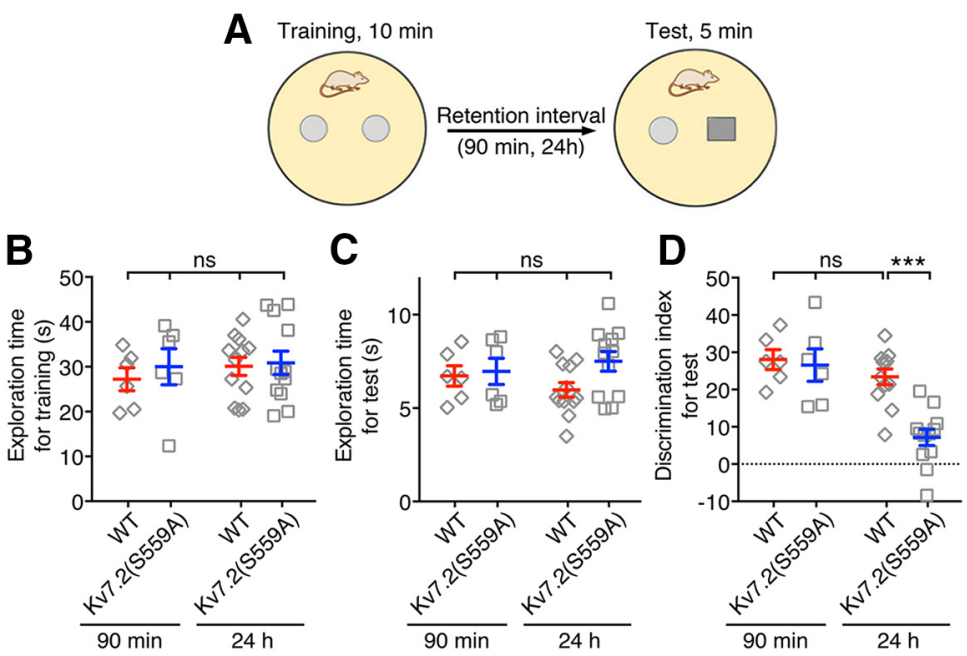

Figure 4. Kv7.2(S559A) mice showed intact short-term retention but impaired long-term retention of object recognition memory. $\boldsymbol{A}$, Schematic drawing illustrating the object recognition test used. $\boldsymbol{B}$, Summary of total exploration time during 10 min training session among short-term retention $(90 \mathrm{~min})$ and long-term retention $(24 \mathrm{~h})$ comparison groups. C, Summary of total exploration time during 5 min test sessions. $\boldsymbol{D}$, Summary of the discrimination index of the object recognition test with short- and long-term retention times. Each data point represents a different animal. Results are shown as the mean \pm SEM. ns, $p>0.05 ;{ }^{* * *} p<0.001$.

exploration of the object in a new location (Fig. 3D; MannWhitney test, $p=0.65)$.

Since spatial memory relies on the hippocampal function (Phillips and LeDoux, 1992; Buzsáki, 2002; Broadbent et al., 2004), we also tested the mice in the contextual fear-conditioning task, which is another test commonly used to evaluate hippocampal function (Anagnostaras et al., 2001; Brown and Aggleton, 2001). In the contextual fear-conditioning paradigm, a single mild footshock $(0.7 \mathrm{~mA}, 2 \mathrm{~s})$ was delivered to a mouse in a chamber with a distinct context, and a freezing response was measured when the mouse was put back into the same chamber $24 \mathrm{~h}$ later (Fig. 3E; Anagnostaras et al., 2001; Maren, 2001; Wiltgen et al., 2006). Kv7.2(S559A) mice showed similar freezing duration as their wild-type littermates when placed into the testing chamber (Fig. 3F; $n=12$ /genotype; Mann-Whitney test, $p=0.19$ ). Normal performance of Kv7.2(S559A) mice in both object location memory and contextual fear-conditioning tasks suggest that $\mathrm{M}$-current suppression does not play a significant role in hippocampus-dependent memory processing.

Kv7.2(S559A) mice had impaired longterm object recognition memory and social odor recognition memory

Next, we tested another type of cognitive function, namely the recognition memory, in Kv7.2(S559A) mice using the object recognition task (Antunes and Biala, 2012; Vogel-Ciernia et al., 2013). In this test, mice were trained for $10 \mathrm{~min}$ with two identical objects in the testing arena and tested with one familiar object replaced with a novel object $90 \mathrm{~min}$ after training for short-term memory or $24 \mathrm{~h}$ after training for long-term memory (Fig. $4 A$ ). Mice in all comparison groups (for $90 \mathrm{~min}, n=6 /$ genotype; for $24 \mathrm{~h}, n=12 /$ genotype) showed similar total exploration time during training (Fig. $4 B$; twoway ANOVA; genotype: $F_{(1,32)}=0.39$, $p=0.54$; treatment: $F_{(1,32)}=0.42, p=0.52$; interaction: $\left.F_{(1,32)}=0.12, p=0.73\right)$, and test sessions (Fig. $4 C$; two-way ANOVA; genotype: $F_{(1,32)}=2.5$, $p=0.12$; treatment: $F_{(1,32)}=0.03, p=0.85$; interaction: $F_{(1,32)}=$ $1.3, p=0.25)$. When tested $90 \mathrm{~min}$ after training, Kv7.2(S559A) and wild-type littermate mice spent significantly longer time on the unfamiliar object, suggesting no deficits in the recognition of objects and short-term object memory (Fig. 4D). However, when the test was conducted $24 \mathrm{~h}$ after the training session, Kv7.2 (S559A) mice spent significantly less time exploring the novel object compared with that of short-term retention tests or that of wild-type mice (Fig. $4 D$; two-way ANOVA; genotype: $F_{(1,32)}=$ $10.2, p=0.003$; treatment: $F_{(1,32)}=18.77, p=0.0001$; interaction: $\left.F_{(1,32)}=7.07, p=0.01\right)$. These results suggest that Kv7.2(S559A) mice have a deficit in long-term retention of object recognition memory. 
Since it has been shown that object recognition memory relies on the function of the perirhinal cortex (Brown and Aggleton, 2001; Suzuki, 2010), we next examined the performance of Kv7.2(S559A) mice in the long-term social odor recognition task, which also requires the perirhinal cortex function (Petrulis and Eichenbaum, 2003; Allen et al., 2007; KholodarSmith et al., 2008; Feinberg et al., 2012). In this test, a mouse was exposed to a wooden bead containing an odor of a stranger mouse, N1, during the training session. At test sessions, the trained mouse was exposed to two wooden beads, one with the same odor from the training session (N1) and another bead carrying the odor of a distinct stranger mouse (N2; Fig. 5A). Because the transition between the perirhinal cortex-independent short-term memory and the perirhinal cortex-dependent long-term memory occurs between $20 \mathrm{~min}$ and $1 \mathrm{~h}$ in the social odor recognition test (Feinberg et al., 2012), we tested $20 \mathrm{~min}$ after training ( $n=6 /$ genotype) for short-term retention and $24 \mathrm{~h}$ after training [ $n=11$ for WT mice and $n=12$ for Kv7.2(S559A) mice] for long-term retention of social odor recognition.

When tested $20 \mathrm{~min}$ after training, Kv7.2(S559A) mice spent more time exploring the wooden bead with the odor of the second stranger mouse (N2; Fig. 5B). However, Kv7.2(S559A) mice lost the preference for the second stranger mouse when tested 24 $\mathrm{h}$ later, while wild-type littermate controls maintained the preference for the second stranger mouse (Fig. 5B; two-way ANOVA; genotype: $F_{(1,31)}=3.4, p=0.07$; treatment: $F_{(1,31)}=4.6, p=0.04$; interaction: $\left.F_{(1,31)}=6.2, p=0.02\right)$. Total exploration times were not different among all test groups (Fig. $5 C$; two-way ANOVA; genotype: $F_{(1,31)}=0.013, p=0.91 ;$ treatment, $F_{(1,31)}=4.0$, $p=0.054$; interaction: $\left.F_{(1,31)}=0.26, p=0.62\right)$.

Because poor performance in odor recognition tasks can be derived from a defective olfactory system, we tested olfactory function with two types of odor-based tasks. The first task examined whether the mice could find hidden food under bedding solely by olfactory cues. Kv7.2(S559A) mice could find food hidden with equivalent latency to that of the wild-type mice $[36.8 \pm 6.8 \mathrm{~s}$ for WT mice $(n=8) ; 37.8 \pm 11.6 \mathrm{~s}$ for Kv7.2(S559A) mice $(n=8)$, Mann-Whitney test, $p=0.67]$. The second task examined whether the mice showed increased exploration of wooden beads with new odors followed by a gradual decrease in exploration time due to habituation. Both wild-type $(n=6)$ and Kv7.2(S559A) mice $(n=6)$ showed a similar increase in exploration when exposed to novel nonsocial and social odors with rapid habituation with repetitive exposure (Fig. 5D; two-way ANOVA; genotype: $F_{(1,10)}=0.06, p=0.82$; treatment: $F_{(14,140)}=$ 15.7, $p<0.0001$; interaction: $\left.F_{(14,140)}=1.1, p=0.36\right)$. These results indicate that Kv7.2(S559A) mice had normal olfactory perception and recognition. In summary, these results suggest that Kv7.2(S559A) mice had normal short-term object/odor recognition memory but had impaired long-term recognition memory.

\section{XE991 restored long-term object recognition memory when administered during the memory consolidation phase}

We reasoned that if a deficit in the memory of Kv7.2(S559A) mice is attributed to attenuated M-current suppression, openchannel inhibition of Kv7 channels should mimic M-current suppression and restore affected memories. XE991 is a unique inhibitor that inhibits neuronal Kv7 channels only in the open conformation (Greene et al., 2017). Therefore, XE991 has negligible effects on silent or inactive neurons that stay below the Kv7 channel activation threshold (Romero et al., 2004; Greene et al.,
A Training, 2 min Test, 2 min

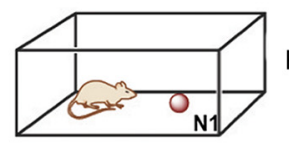
$\stackrel{\text { Retention interval }}{\longrightarrow}$ (20 min, 24 h)

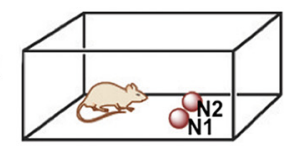

B

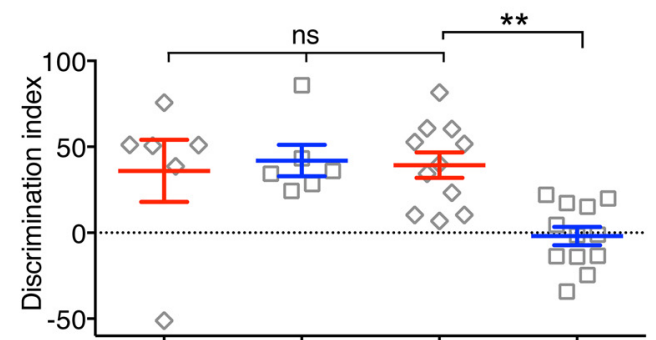

C

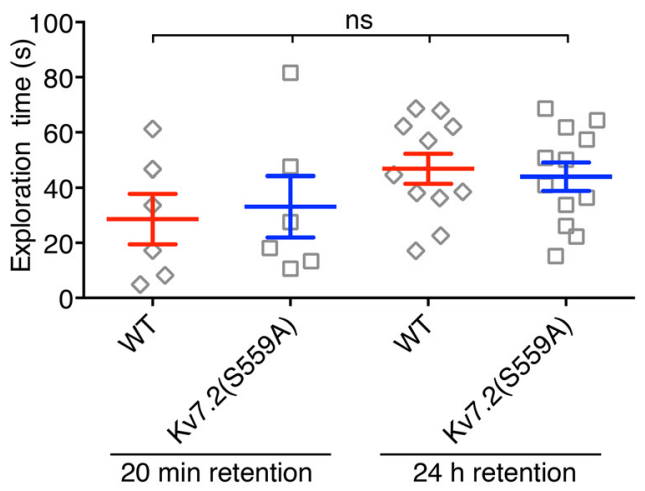

D

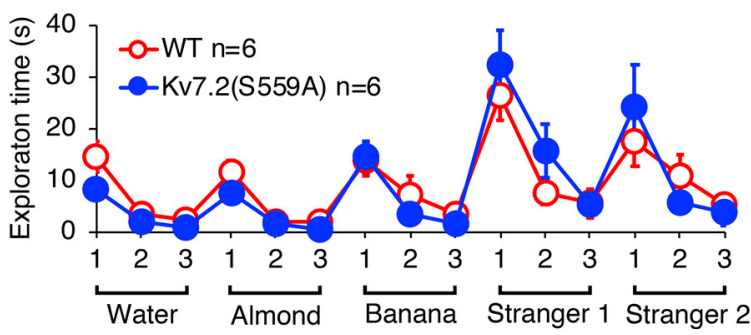

Figure 5. Kv7.2(S559A) mice had impaired long-term retention of social odor recognition memory. $\boldsymbol{A}$, Schematic drawing illustrating the social odor recognition test. $\boldsymbol{B}$, Summary of short-term (20 $\mathrm{min})$ and long-term $(24 \mathrm{~h})$ retention of social odor recognition memory in WT and Kv7.2(S559A) mice. C, Summary of total exploration times during test sessions. Each data point represents a different animal. $\boldsymbol{D}$, Summary of olfactory function test. Results are shown as the mean \pm SEM. ns, $p>0.05 ;{ }^{* *} p<0.01$.

2017). If facilitation of neuronal firing mediated by M-current modulation is required for memory processing, open-channel inhibition of Kv7.2(S559A) channels in involved neurons should compensate $\mathrm{M}$-current suppression and restore the process (Fig. $6 A)$. The administration of XE991 $(2 \mathrm{mg} / \mathrm{kg})$ did not change exploration behavior during training (Fig. $6 B-D$; WT mice: veh, $n=6$; XE991, $n=7$; Kv7.2(S559A) mice: veh, $n=7$; XE991, $n=8$; two-way ANOVA, $p>0.05$ ) or test sessions (Fig. $6 E-G$; two-way ANOVA, $p>0.05)$. For object recognition memory, XE991 $(2 \mathrm{mg} / \mathrm{kg})$ administration at pre-training improved the performance of Kv7.2(S559A) mice in the object recognition task with a $24 \mathrm{~h}$ retention equivalent to that of the wild-type littermate controls (Fig. $6 H$; two-way ANOVA; genotype: $F_{(1,24)}=19.45$, $p=0.0002$; treatment: $F_{(1,24)}=5.6, p=0.026$; interaction: $F_{(1,24)}=$ $2.11, p=0.16)$. Performance of the wild-type littermate controls 
A
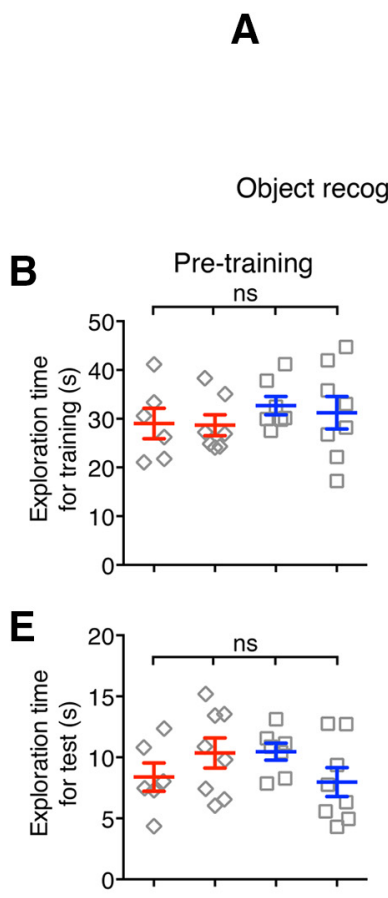

H

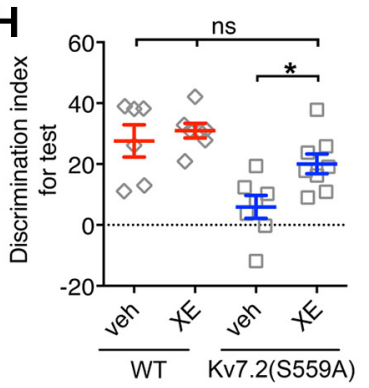

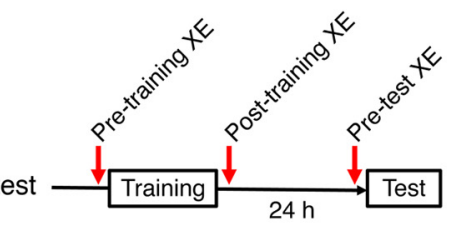
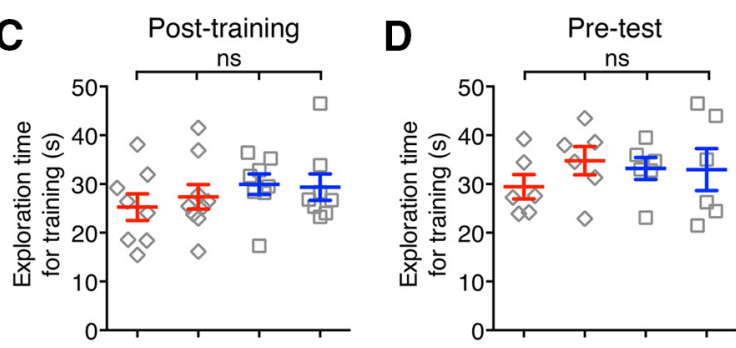

$\mathbf{F}$
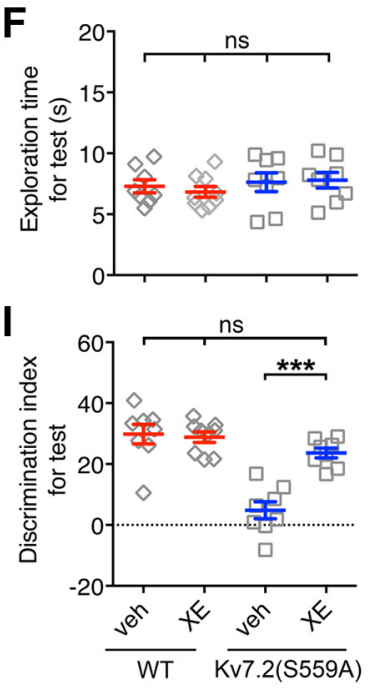

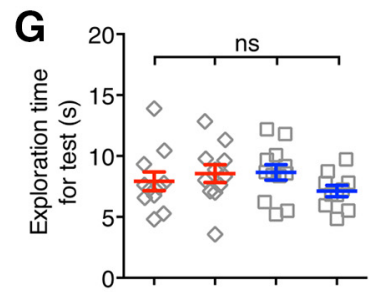

$\mathbf{J}$

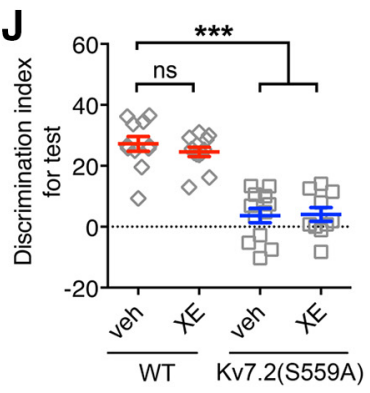

Figure 6. Pharmacological rescue of long-term retention of object recognition memory by XE991 and its effective time window. $\boldsymbol{A}$, Schematic drawing showing three types of administration schedules of XE991 (XE; $2 \mathrm{mg} / \mathrm{kg}$ ) during object recognition tests. $\boldsymbol{B}$ D, Summary of total exploration times during training sessions for WT and Kv7.2(S559A) mice with injection of PBS veh control or XE991 at pre-training $(\boldsymbol{B})$, post-training $(\boldsymbol{C})$, or pre-test $(\boldsymbol{D})$. $\boldsymbol{E}-\mathbf{G}$, Summary of total exploration times during test sessions of WT and Kv7.2(S559A) mice with the injection of veh control or XE991 at pre-training (E), post-training (F), or pre-test $(\boldsymbol{G})$. $\boldsymbol{H}-\boldsymbol{J}$, Performance in novel object recognition task as discrimination indexes in WT and Kv7.2(S559A) mice with injection of veh control or XE991 at pre-training $(\boldsymbol{H})$, post-training $(\boldsymbol{I})$, or pre-test $(\boldsymbol{J})$. Each data point represents a different animal. Results are shown as the mean \pm SEM. ${ }^{* * *} p<0.001 ;{ }^{*} p<0.05 ; \mathrm{ns}, p>0.05$.

was not affected by this dose of XE991 (Fig. 6H). Since the administration of XE991 before training may affect both the acquisition as well as the consolidation phase of memory formation, we tested the effect of XE991 administration immediately after training (post-training) to test its effect on memory consolidation phase alone (Fig. 6F,I). The administration of XE991 post-training restored long-term object recognition memory inKv7.2(S559A) mice (Fig. 6I; two-way ANOVA; genotype: $F_{(1,28)}=36.8, p<0.0001$; treatment: $F_{(1,28)}=12.7, p=0.0014$; interaction: $\left.F_{(1,28)}=16.7, p=0.0003\right)$. We also tested the effect of XE991 on memory retrieval by administration of XE991 $15 \mathrm{~min}$ before the test session (pre-test; Fig. 6G,J). Pre-test administration of XE991 did not improve the performance of Kv7.2(S559A) mice (Fig. 6J; two-way ANOVA; genotype: $F_{(1,41)}=102.6$, $p<0.0001$; treatment: $F_{(1,41)}=0.28, p=0.60$; interaction: $F_{(1,41)}$ $=0.48, p=0.49$ ). Performance of the wild-type littermate controls was not affected regardless of the timing of XE991 administration (Fig. 6H-J; two-way ANOVA $p>0.05$ ). We concluded that $\mathrm{M}$-current suppression is important for consolidation of object recognition memory.
Training triggered intact c-Fos induction in the hippocampus but reduced c-Fos induction in the perirhinal cortex in Kv7.2(S559A) mice

Because Kv7.2(S559A) mice showed intact object location memory and impaired object recognition memory, we questioned whether there were differences in neuronal activation between the hippocampus and the perirhinal cortex. To address this question, we used c-Fos induction as an indicator of highly activated neurons (Bullitt, 1990; Guzowski et al., 2005). Since training sessions for object location task and object recognition task are identical, the induction of c-Fos can be compared in these two brain regions in the same mouse. Mouse brains were collected $2 \mathrm{~h}$ after training sessions $(n=8$ per genotype, $n=9$ for home cage control) and c-Fos induction was detected by immunostaining (Fig. 7). In the hippocampus, the number of c-Fos-induced cells were increased to a similar extent in both genotypes (Fig. 7A,B; two-way ANOVA; genotype: $F_{(1,30)}=0.21, p=0.65$; treatment: $F_{(1,30)}=94.95, p<0.0001$; interaction: $\left.F_{(1,30)}=0.002, p=0.97\right)$. In addition, post-training administration of XE991 had no further effects on c-Fos induction (Fig. 7C; $n=6 /$ group, two-way ANOVA, $p>$ $0.05)$.

In contrast, c-Fos induction in the perirhinal cortex of Kv7.2(S559A) mice was significantly lower than that of wild-type control mice after training (Fig. $7 D, E$; two-way ANOVA; genotype: $F_{(1,30)}=20.5, p<0.0001$; treatment: $F_{(1,30)}=111.0, p<0.0001$; interaction: $\left.F_{(1,30)}=23.7, p<0.0001\right)$. Similar to our behavioral results, post-training administration of XE991 restored c-Fos induction in the perirhinal cortex of Kv7.2(S559A) mice to the normal level (Fig. $7 F$; two-way ANOVA; genotype: $F_{(1,20)}=1.07, p=0.31$; treatment: $F_{(1,20)}=14.37, p=0.001$; interaction: $F_{(1,20)}=6.1$, $p=0.02$ ). These results suggest that $\mathrm{M}$-current suppression plays an important role in the regulation of post-training firing of neuronal circuits involving the perirhinal cortex during memory consolidation but not in the hippocampus.

\section{Discussion}

We demonstrated that neurons from Kv7.2(S559A) mice showed not only attenuated M-current suppression, but also a lack of neuronal hyperexcitability when muscarinic receptors were stimulated. Since the discovery of the M-current, it has been well established that M-current suppression correlates well with the increase in neuronal excitation induced by muscarinic stimulation (Brown and Adams, 1980; Delmas and Brown, 2005). 
A Hippocampus
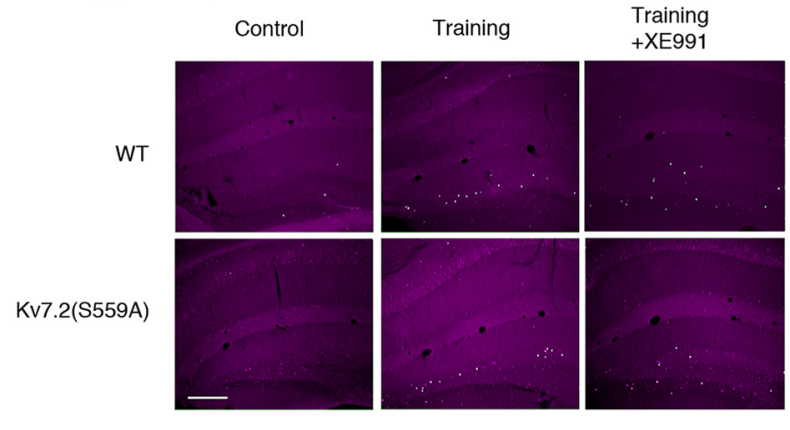

B

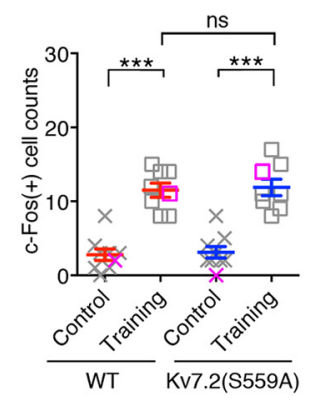

C

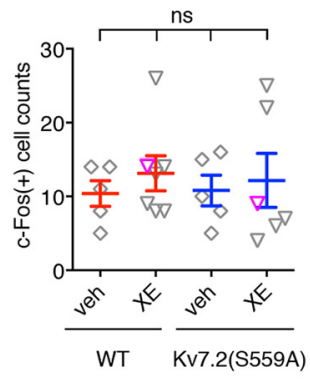

E

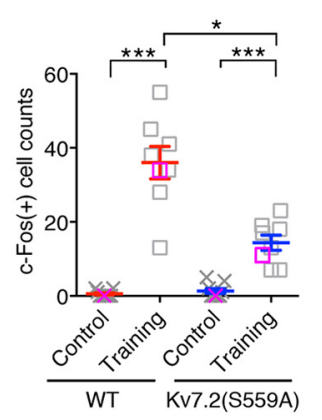

$\mathbf{F}$

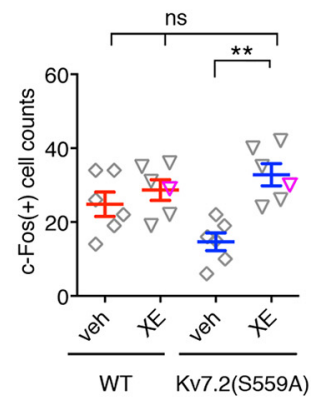

D Perirhinal cortex

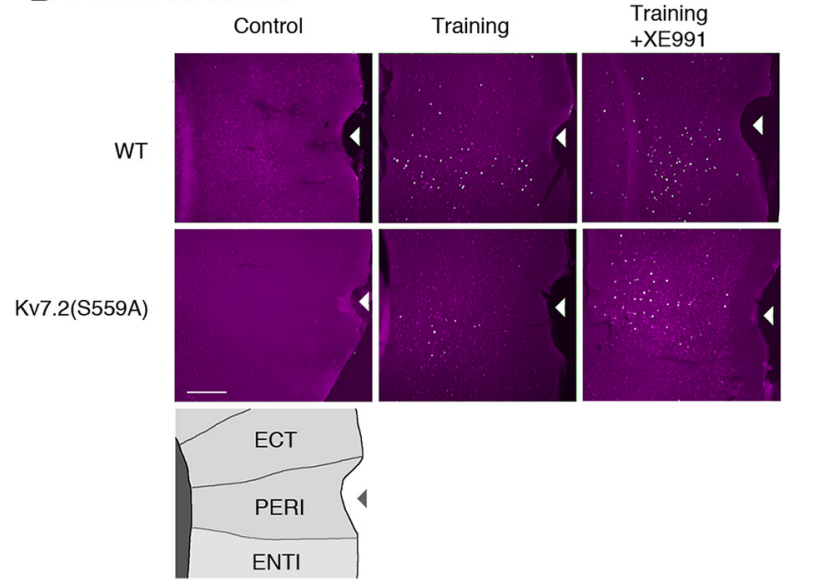

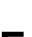


projects to areas including the hippocampus and the entorhinal cortex, and another category projects to areas including olfactory areas, medial prefrontal cortex, and the perirhinal cortex (Li et al., 2018). Interestingly, projections to the hippocampus and the perirhinal cortex are mediated by distinct groups of neurons (Li et al., 2018). Distinct combinations of hyperexcitability in multiple brain regions mediated by M-current suppression would provide distinct contribution to different aspects of cognitive processes.

XE991 and its prototypical analog linopirdine were originally discovered as cognitive enhancers before they were identified as Kv7 channel inhibitors (DeNoble et al., 1990; Brioni et al., 1993). Linopirdine has been shown to improve experimental amnesias (DeNoble et al., 1990; Brioni et al., 1993; Fontana et al., 1994). Interestingly, linopirdine improves cognitive impairment caused by hypoxia or cholinergic dysfunction, but does not enhance cognition in healthy animals (Fontana et al., 1994; Flagmeyer and Van Der Staay, 1995). This difference may be attributed to open-channel inhibition of Kv7 channel, which can enhance impaired neuronal activity to a level sufficient for processing information, while having no effects in healthy brains because neuronal activity is already in an optimal range.

In summary, we identified a key role for M-current suppression in memory consolidation of object recognition memory. In contrast, M-current suppression appeared to not be required for hippocampus-dependent memory formation. Further studies will be necessary to determine the extent of what types of memory processing M-current suppression is involved in, and how exactly $\mathrm{M}$-current suppression is regulating gene expression that is required for memory consolidation. Overall, the results shown here suggest a highly specific role for M-current suppression in specific forms of memory formation.

\section{References}

Allen TA, Furtak SC, Brown TH (2007) Single-unit responses to $22 \mathrm{kHz}$ ultrasonic vocalizations in rat perirhinal cortex. Behav Brain Res 182:327336.

Anagnostaras SG, Gale GD, Fanselow MS (2001) Hippocampus and contextual fear conditioning: recent controversies and advances. Hippocampus 11:8-17.

Antunes M, Biala G (2012) The novel object recognition memory: neurobiology, test procedure, and its modifications. Cogn Process 13:93-110.

Balderas I, Rodriguez-Ortiz CJ, Salgado-Tonda P, Chavez-Hurtado J, McGaugh JL, Bermudez-Rattoni F (2008) The consolidation of object and context recognition memory involve different regions of the temporal lobe. Learn Mem 15:618-624.

Brioni JD, Curzon P, Buckley MJ, Arneric SP, Decker MW (1993) Linopirdine (DuP996) facilitates the retention of avoidance training and improves performance of septal-lesioned rats in the water maze. Pharmacol Biochem Behav 44:37-43.

Broadbent NJ, Squire LR, Clark RE (2004) Spatial memory, recognition memory, and the hippocampus. Proc Natl Acad Sci U S A 101:1451514520.

Brown DA, Adams PR (1980) Muscarinic suppression of a novel voltage-sensitive $\mathrm{K}+$ current in a vertebrate neurone. Nature 283:673-676.

Brown MW, Aggleton JP (2001) Recognition memory: what are the roles of the perirhinal cortex and hippocampus? Nat Rev Neurosci 2:51-61.

Bullitt E (1990) Expression of c-fos-like protein as a marker for neuronal activity following noxious stimulation in the rat. J Comp Neurol 296:517530.

Buzsáki G (2002) Theta oscillations in the hippocampus. Neuron 33:325340.

Delmas P, Brown DA (2005) Pathways modulating neural KCNQ/M (Kv7) potassium channels. Nat Rev Neurosci 6:850-862.

Dennis SH, Pasqui F, Colvin EM, Sanger H, Mogg AJ, Felder CC, Broad LM, Fitzjohn SM, Isaac JT, Mellor JR (2016) Activation of muscarinic M1 acetylcholine receptors induces long-term potentiation in the hippocampus. Cereb Cortex 26:414-426.
DeNoble VJ, DeNoble KF, Spencer KR, Johnson LC, Cook L, Myers MJ, Scribner RM (1990) Comparison of DuP 996, with physostigmine, THA and 3,4-DAP on hypoxia-induced amnesia in rats. Pharmacol Biochem Behav 36:957-961.

Duangdao DM, Clark SD, Okamura N, Reinscheid RK (2009) Behavioral phenotyping of neuropeptide S receptor knockout mice. Behav Brain Res 205:1-9.

Feinberg LM, Allen TA, Ly D, Fortin NJ (2012) Recognition memory for social and non-social odors: differential effects of neurotoxic lesions to the hippocampus and perirhinal cortex. Neurobiol Learn Mem 97:7-16.

Flagmeyer I, Van Der Staay FJ (1995) Linopiridine (DUP 996; AVIVA): its effects in the Morris water escape tank and on retention of an incompletely acquired bar-press response in rodents. Pharmacol Biochem Behav 51:111-117.

Fontana DJ, Inouye GT, Johnson RM (1994) Linopirdine (DuP 996) improves performance in several tests of learning and memory by modulation of cholinergic neurotransmission. Pharmacol Biochem Behav 49:1075-1082.

Greene DL, Hoshi N (2017) Modulation of Kv7 channels and excitability in the brain. Cell Mol Life Sci 74:495-508.

Greene DL, Kang S, Hoshi N (2017) XE991 and Linopirdine Are StateDependent Inhibitors for Kv7/KCNQ Channels that Favor Activated Single Subunits. J Pharmacol Exp Ther 362:177-185.

Greene DL, Kosenko A, Hoshi N (2018) Attenuating M-current suppression in vivo by a mutant Kcnq2 gene knock-in reduces seizure burden and prevents status epilepticus-induced neuronal death and epileptogenesis. Epilepsia 59:1908-1918.

Guzowski JF, Timlin JA, Roysam B, McNaughton BL, Worley PF, Barnes CA (2005) Mapping behaviorally relevant neural circuits with immediateearly gene expression. Curr Opin Neurobiol 15:599-606.

Hasselmo ME (2006) The role of acetylcholine in learning and memory. Curr Opin Neurobiol 16:710-715.

Hoshi N, Zhang JS, Omaki M, Takeuchi T, Yokoyama S, Wanaverbecq N, Langeberg LK, Yoneda Y, Scott JD, Brown DA, Higashida H (2003) AKAP150 signaling complex promotes suppression of the M-current by muscarinic agonists. Nat Neurosci 6:564-571.

Hoshi N, Langeberg LK, Scott JD (2005) Distinct enzyme combinations in AKAP signalling complexes permit functional diversity. Nat Cell Biol 7:1066-1073.

Hoshi N, Langeberg LK, Gould CM, Newton AC, Scott JD (2010) Interaction with AKAP79 modifies the cellular pharmacology of PKC. Mol Cell 37:541-550

Jentsch TJ (2000) Neuronal KCNQ potassium channels: physiology and role in disease. Nat Rev Neurosci 1:21-30.

Kaech S, Banker G (2006) Culturing hippocampal neurons. Nat Protoc 1:2406-2415.

Kay HY, Greene DL, Kang S, Kosenko A, Hoshi N (2015) M-current preservation contributes to anticonvulsant effects of valproic acid. J Clin Invest 125:3904-3914.

Kholodar-Smith DB, Allen TA, Brown TH (2008) Fear conditioning to discontinuous auditory cues requires perirhinal cortical function. Behav Neurosci 122:1178-1185

Kosenko A, Kang S, Smith IM, Greene DL, Langeberg LK, Scott JD, Hoshi N (2012) Coordinated signal integration at the M-type potassium channel upon muscarinic stimulation. EMBO J 31:3147-3156.

Kremin T, Gerber D, Giocomo LM, Huang SY, Tonegawa S, Hasselmo ME (2006) Muscarinic suppression in stratum radiatum of CA1 shows dependence on presynaptic M1 receptors and is not dependent on effects at GABA(B) receptors. Neurobiol Learn Mem 85:153-163.

Kwapis JL, Alaghband Y, López AJ, Long JM, Li X, Shu G, Bodinayake KK, Matheos DP, Rapp PR, Wood MA (2019) HDAC3-mediated repression of the $\mathrm{Nr} 4 \mathrm{a}$ family contributes to age-related impairments in long-term memory. J Neurosci 39:4999-5009.

Li X, Yu B, Sun Q, Zhang Y, Ren M, Zhang X, Li A, Yuan J, Madisen L, Luo Q, Zeng H, Gong H, Qiu Z (2018) Generation of a whole-brain atlas for the cholinergic system and mesoscopic projectome analysis of basal forebrain cholinergic neurons. Proc Natl Acad Sci U S A 115:415-420.

Maren S (2001) Neurobiology of Pavlovian fear conditioning. Annu Rev Neurosci 24:897-931.

Miceli F, Soldovieri MV, Joshi N, Weckhuysen S, Cooper E, Taglialatela M (2010) KCNQ2-related disorders. In: GeneReviews (Adam MP, Ardinger 
HH, Pagon RA, Wallace SE, Bean LJH, Stephens K, Amemiya A, eds). Seattle, WA: University of Washington.

Miceli F, Soldovieri MV, Joshi N, Weckhuysen S, Cooper ECTaglialatela M (2014) KCNQ3-Related Disorders. In: GeneReviews (Adam MP, Ardinger HH, Pagon RA, Wallace SE, Bean LJH, Stephens K, Amemiya A, eds). Seattle, WA: University of Washington.

Okamura N, Habay SA, Zeng J, Chamberlin AR, Reinscheid RK (2008) Synthesis and pharmacological in vitro and in vivo profile of 3-oxo-1,1diphenyl-tetrahydro-oxazolo[3,4-a]pyrazine-7-carboxylic acid 4-fluorobenzylamide (SHA 68), a selective antagonist of the neuropeptide $\mathrm{S}$ receptor. J Pharmacol Exp Ther 325:893-901.

Petrulis A, Eichenbaum H (2003) The perirhinal-entorhinal cortex, but not the hippocampus, is critical for expression of individual recognition in the context of the Coolidge effect. Neuroscience 122:599-607.

Phillips RG, LeDoux JE (1992) Differential contribution of amygdala and hippocampus to cued and contextual fear conditioning. Behav Neurosci 106:274-285.

Picciotto MR, Higley MJ, Mineur YS (2012) Acetylcholine as a neuromodulator: cholinergic signaling shapes nervous system function and behavior. Neuron 76:116-129.

Romero M, Reboreda A, Sánchez E, Lamas JA (2004) Newly developed blockers of the M-current do not reduce spike frequency adaptation in cultured mouse sympathetic neurons. Eur J Neurosci 19:2693-2702.
Seeger T, Fedorova I, Zheng F, Miyakawa T, Koustova E, Gomeza J, Basile AS, Alzheimer C, Wess J (2004) M2 muscarinic acetylcholine receptor knock-out mice show deficits in behavioral flexibility, working memory, and hippocampal plasticity. J Neurosci 24:10117-10127.

Suzuki WA (2010) Untangling memory from perception in the medial temporal lobe. Trends Cogn Sci 14:195-200.

Urban DJ, Roth BL (2015) DREADDs (designer receptors exclusively activated by designer drugs): chemogenetic tools with therapeutic utility. Annu Rev Pharmacol Toxicol 55:399-417.

Vogel-Ciernia A, Wood MA (2014) Examining object location and object recognition memory in mice. Curr Protoc Neurosci 69:8.31.1-17.

Vogel-Ciernia A, Matheos DP, Barrett RM, Kramár EA, Azzawi S, Chen Y, Magnan CN, Zeller M, Sylvain A, Haettig J, Jia Y, Tran A, Dang R, Post RJ, Chabrier M, Babayan AH, Wu JI, Crabtree GR, Baldi P, Baram TZ, Lynch G, Wood MA (2013) The neuron-specific chromatin regulatory subunit BAF53b is necessary for synaptic plasticity and memory. Nat Neurosci 16:552-561.

Wiltgen BJ, Sanders MJ, Anagnostaras SG, Sage JR, Fanselow MS (2006) Context fear learning in the absence of the hippocampus. J Neurosci 26:5484-5491.

Xu YL, Reinscheid RK, Huitron-Resendiz S, Clark SD, Wang Z, Lin SH, Brucher FA, Zeng J, Ly NK, Henriksen SJ, de Lecea L, Civelli O (2004) Neuropeptide S: a neuropeptide promoting arousal and anxiolytic-like effects. Neuron 43:487-497. 